Check for updates

Cite this: RSC Med. Chem., 2020, 11, 876

Received 9th May 2020

Accepted 19th June 2020

DOI: $10.1039 / d 0 m d 00154 f$

rsc.li/medchem

\title{
Covalent inhibitors: a rational approach to drug discovery
}

\author{
Fandi Sutanto, Markella Konstantinidou (iD) and Alexander Dömling (D) *
}

Covalent inhibitors are recognized as an important component in drug discovery and therapeutics. Since the first appearance of covalent inhibitors in the late 18th century, the field has advanced significantly and currently about $30 \%$ of the marketed drugs are covalent inhibitors. The numerous advantages of covalent inhibitors are counteracting the initial concerns regarding potential off-target toxicity. Thus, continuous research, especially for cancer targets is reported. The aim of this review is to provide a short historic overview and focus on recently developed covalent inhibitors (2011-2019), including structural aspects and examples on challenging targets.

\section{Introduction}

Traditionally, drug design of small molecules is based on their ability to interact with their biological targets under equilibrium binding states. These binding states occur in a fast and reversible process, which affects the duration of the therapeutic response. Thus, prolongation of therapeutic response can be extended by increasing the duration of interaction between a drug and its target. This type of prolonged interaction can be achieved by covalent inhibitors. Covalent inhibitors bind to their target in two steps, starting

Department of Pharmacy, Group of Drug Design, University of Groningen, A.

Deusinglaan 1, 9713 AV, Groningen, The Netherlands.

E-mail: a.s.s.domling@rug.nl; Web: http://www.drugdesign.nl with equilibrium bond formation, and ending with covalent bond formation. The final state is considered to be irreversible, resulting in a drug-protein complex that is different from that formed with a normal equilibrium bond (Fig. 1).

Covalent inhibitors in general are compounds that by design are intended to form a covalent bond with a specific molecular target. The covalent bond can be either reversible or irreversible, depending on the chosen warhead. A number of different warheads have been exploited to target specific amino acid residues, including among others cysteine, serine, threonine, tyrosine and lysine. A detailed overview of covalent warheads, as well as the amino acids that they target was recently published. ${ }^{1}$

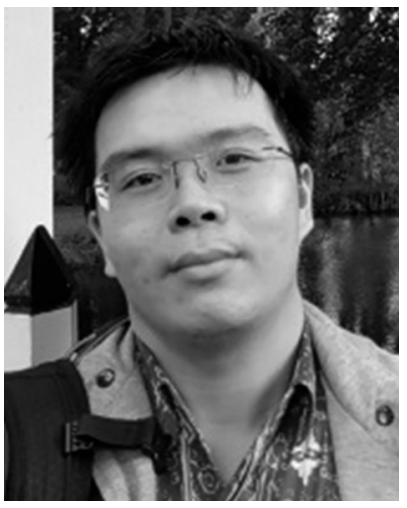

Fandi Sutanto
Fandi Sutanto obtained his Pharmacist Degree (2011) and his M. Sc. in Pharmacochemistry (2013) from the Bandung Institute of Technology, Indonesia. He then joined an analytical department in a pharmaceutical industry as a scientist. Later on, he started his $P h D$ journey in 2016 under the guidance of Prof. A. Dömling in the Drug Design Group in the University of Groningen, The Netherlands. His PhD research focuses on the development of covalent inhibitors utilizing the multicomponent reactions.

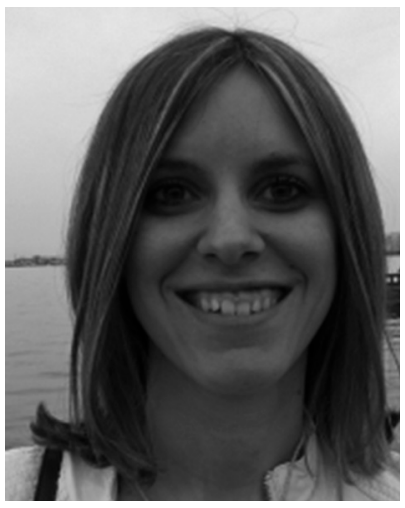

Markella Konstantinidou
Markella Konstantinidou obtained her Degree in Pharmacy (2012) and her M.Sc. in Medicinal Chemistry (2014) from the Aristotle University of Thessaloniki, Greece. Afterwards, she joined the National Hellenic Research Foundation in Athens, as a research assistant. In 2016, she joined as a PhD candidate in the Drug Design Group in the University of Groningen in The Netherlands under the guidance of Prof. A. Dömling. She received her PhD in February 2020. Her PhD research included multicomponent reactions, medicinal chemistry applications and new modalities. 


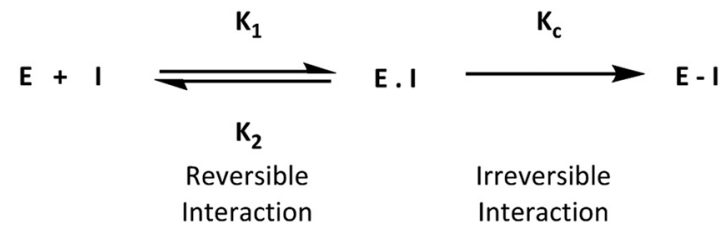

Fig. 1 General mechanism of covalent interaction between a small molecule and its target.

Despite the initial skepticism of pharmaceutical industry to develop covalent inhibitors, in the last 50 years, the development of covalent inhibitors has shown a significant increase (Fig. 2). ${ }^{2,3}$ Numerous drug candidates are progressing through clinical trials or being approved by the FDA. Today, there are at least 50 FDA-approved drugs that act as covalent inhibitors.

Due to the growing interest in covalent inhibitors as drugs, it is worthwhile to examine the design of these drugs in detail. In this review, after a short historic overview, the advantages and disadvantages of covalent inhibitors are discussed. Then, we focus on covalent inhibitors that reached the market or are in advanced clinical trials (2011-2019). In the last part, we review recent applications of covalent inhibitors.

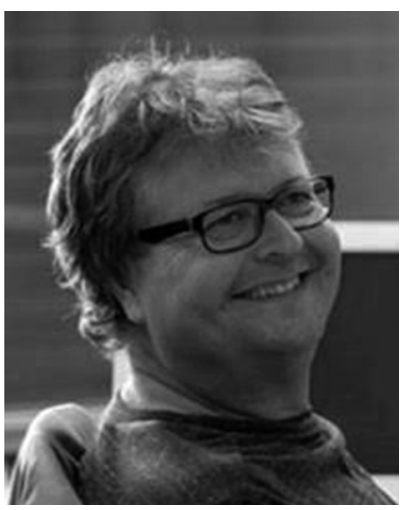

Alexander Dömling
Prof. Alexander Dömling has held the chair for Drug Design at the University of Groningen since 2011. He studied chemistry and biology at the Technische Universität München and obtained his Ph.D. under the guidance of Ivar Ugi. After a postdoc under a Humboldt Fellowship in the group of the Nobel Laureate Barry Sharpless, he founded the biotech company Morphochem and later Carmolex Inc. After his habilitation he worked as full professor at the University of Pittsburgh in the School of Pharmacy. His interests are centered around multicomponent reaction (MCR) chemistry and its application to problems in drug design. His special focus is on MCR-centered pharmacophore methods, structure-based drug design and MCRcentered fragment-based drug design. A creto of his lab is automation + miniaturization = acceleration. He is the author of more than 270 scientific articles, reviews and book contributions. He has applied for more than 50 patents. His long-term vision is to bring a novel drug to patients in an indicated area of unmet medical need.

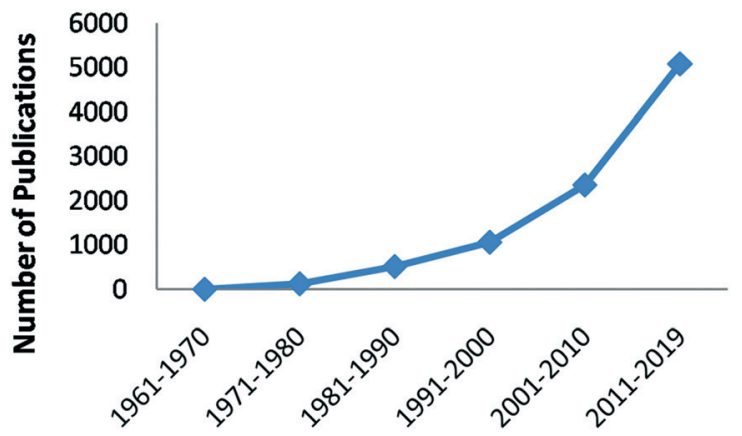

Fig. 2 Number of publications per decade obtained from a search of the term "covalent drug" in SciFinder®.

\section{Historic background of covalent inhibitors}

The earliest covalent inhibitor to be introduced was acetylsalicylic acid (aspirin), which has been marketed since 1899 (Fig. 3). The acetyl warhead of aspirin acetylates Ser-530 of prostaglandin endoperoxide (PGH) synthase-1, inactivating the cyclooxygenase activity of the enzyme. ${ }^{4}$ Penicillin, which was discovered in 1928 , also acts as a covalent inhibitor, as the $\beta$-lactam binds covalently to the active site serine (Ser-36) of the bacterial enzyme DD-transpeptidase to form penicilloylenzyme which is inactive, thus making it incapable of synthesizing the cell-wall. ${ }^{5}$

In 1975, timoprazole showed inhibition towards gastric acid secretion. ${ }^{6}$ However, due to its toxicity, the development of timoprazole was stopped. Its derivative, omeprazole, was discovered in 1979, as the first class of drug which is now known as proton pump inhibitor (PPI). ${ }^{7,8}$ Omeprazole forms a disulfide bond with the sulfhydryl group of the hydrogenpotassium ATPase. The second of the PPI drugs to reach the market was lansoprazole, which was patented in 1984, launched in early 1990s, and approved by FDA in 1995.

In the 1990s, two examples of the most successful covalent drugs were fosfomycin and clopidogrel. Fosfomycin was found in $1969,{ }^{9}$ however, the approval and medical usage started in 1996. The drug inhibits bacterial cell wall biogenesis through inactivation of the enzyme MurA. ${ }^{10}$ It bears an epoxide warhead, which is targeting the active site residue Cys-115 of MurA. Clopidogrel was patented in 1982, and approved for medical use in 1997. Clopidogrel is a prodrug, which is used as antiplatelet medication. The prodrug undergoes a two-step metabolic activation; the thiophene ring is first oxidized towards the inactive in vitro 2-oxo-clopidogrel and then by ring opening, the active thiolcarboxylic acid metabolite is released. The latter binds irreversibly via the $-\mathrm{SH}$ group to the $\mathrm{P}_{2} \mathrm{Y}_{21}$ purinergic receptor, thus preventing binding of $\mathrm{ADP}$ to the $\mathrm{P}_{2} \mathrm{Y}_{21}$ receptor. Bortezomib, one of the few covalent inhibitors with boronic acid warhead, is an anticancer medication. It was approved and marketed in 2003. In 2009, a drug containing nitrile warhead saxagliptin was developed. Saxagliptin is an antidiabetic drug which was used to treat type 2 diabetes 


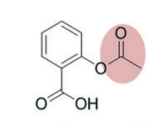

Synthesis of Aspirin by Bayer

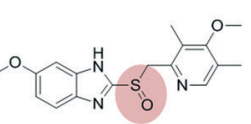

Omeprazole (1979), first class proton pump inhibitor

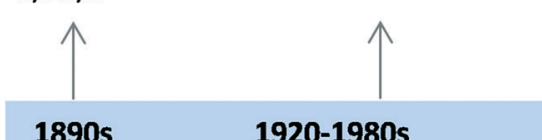

1890s

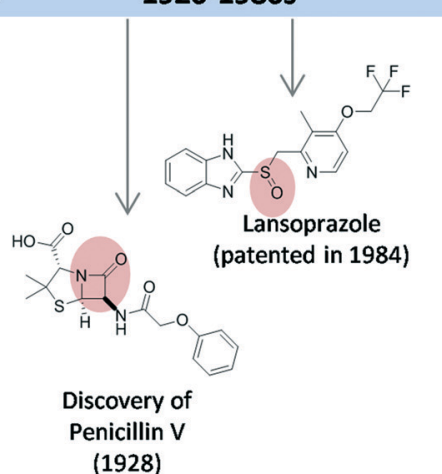

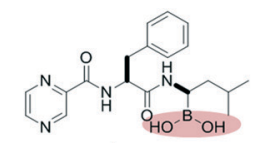

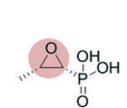

Fosfomycin, anti infective approved 1996
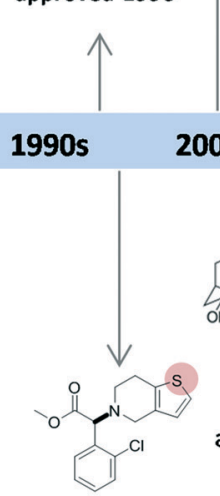

Clopidogrel antiplatelet approved 1997

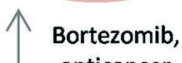
anticancer approved 2003
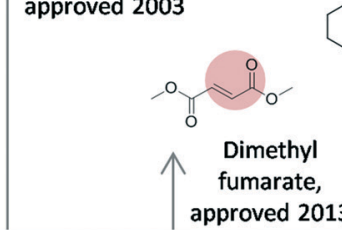

Dacomitinib, inhibitor of EGFR

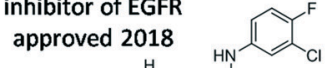
$\overbrace{}^{2}$
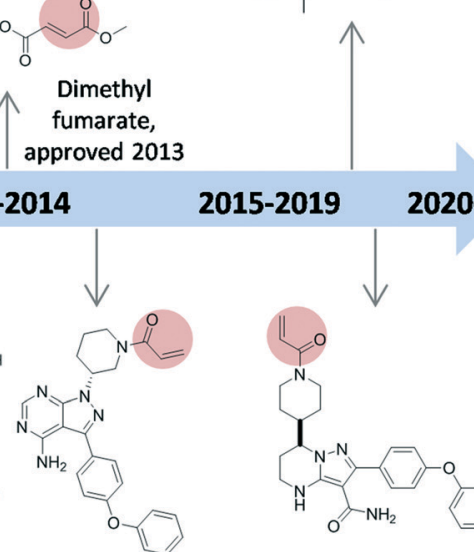

2015-2019

2020-

Ibrutinib,

BTK inhibitor approved 2014

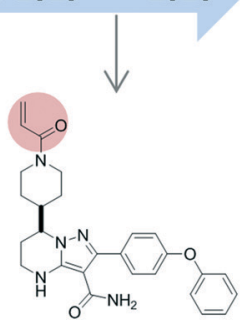

Zanubrutinib,

BTK inhibitor

approved 2019

Fig. 3 A historic overview of covalent inhibitors and their approval dates. The warheads are highlighted in red.

mellitus, succeeding vildagliptin which was withdrawn in 2008. Today, there are at least 50 approved covalent inhibitors used to treat ailments ranging from obesity to cancer. ${ }^{3}$ Zanubrutinib (approved November 2019, brand name Brukinsa ${ }^{\circledR}$ ) and dacomitinib (approved September 2018, marketed under the name Vizimpro®) are two of the most recently approved covalent inhibitors of BTK and EGFR, respectively.

\section{Advantages and disadvantages of covalent inhibitors}

Toxicity and efficacy are the main reasons for the attrition of drug candidates during clinical study. ${ }^{11}$ In these regards, covalent inhibitors have several advantages: (1) improving efficiency, (2) lowering the dose, (3) increasing compliance

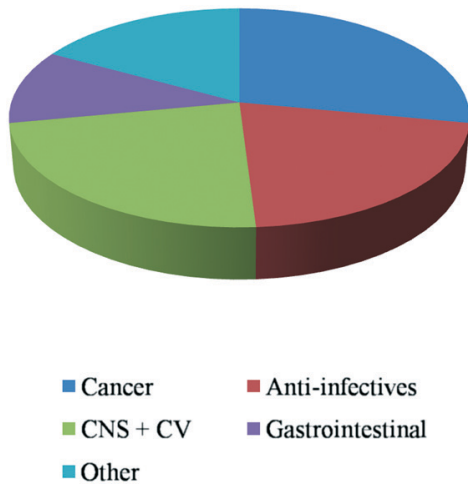

Fig. 4 Approved covalent drugs by therapeutic indication.
Table 1 FDA-approved covalent drugs (2011-2019)

\begin{tabular}{lllll}
\hline & & Therapeutic & & \\
No & Name & area & Warhead & Year \\
\hline 1. & Telaprevir & Anti-HCV & $\alpha$-Ketoamide & 2011 \\
2. & Boceprevir & Anti-HCV & $\alpha$-Ketoamide & 2011 \\
3. & Abiraterone & Anticancer & - & 2011 \\
4. & Afatinib & Anticancer & $\alpha, \beta$-Unsaturated carbonyl & 2013 \\
5. & Dimethyl & Multiple & $\alpha, \beta$-Unsaturated carbonyl & 2013 \\
& fumarate & sclerosis & & \\
6. & Ibrutinib & Anticancer & $\alpha, \beta$-Unsaturated carbonyl & 2014 \\
7. & Osimertinib & Anticancer & $\alpha, \beta$-Unsaturated carbonyl & 2015 \\
8. & Olmutinib & Anticancer & $\alpha, \beta$-Unsaturated carbonyl & 2015 \\
9. & Narlaprevir & Anti-HCV & $\alpha$-Ketoamide & 2016 \\
10. & Acalabrutinib & Anticancer & $\alpha, \beta$-Unsaturated & 2017 \\
& & & propargylamide & \\
11. Neratinib & Anticancer & $\alpha, \beta$-Unsaturated carbonyl & 2017 \\
12. & Dacomitinib & Anticancer & $\alpha, \beta$-Unsaturated carbonyl & 2018 \\
13. & Selinexor & Anticancer & $\alpha, \beta$-Unsaturated carbonyl & 2019 \\
14. & Zanubrutinib & Anticancer & $\alpha, \beta$-Unsaturated carbonyl & 2019 \\
& & & &
\end{tabular}
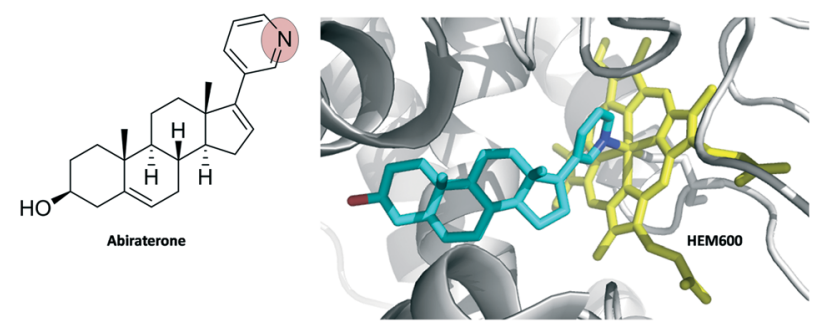

Fig. 5 Abiraterone complex with human cytochrome P450 CYP17A1 (PDB ID 3RUK). Abiraterone is shown as cyan sticks and HEM-600 is shown as yellow sticks. 
(a)<smiles>CCCC(CC)(NC(=O)[C@@H]1[C@@H]2CCC[C@H]2CN1C(=O)[C@@H](NC(=O)C(NC(=O)c1cnccn1)C1CCCCC1)C(C)(C)C)C(=O)C(=O)NC1CC1</smiles>

(b)<smiles>CCCC[C@]1(NC(=O)C2CC3(C)CC2CN3C(=O)[C@@H](NC(=O)NC2(CS(=O)(=O)C(C)(C)C)CCCCC2)C(C)(C)C)CCCCC1(C)C</smiles>

Narlaprevir

(c)<smiles>CC(C)(C)NC(=O)NC(C(=O)N1CC2[C@H]([C@H]1C(=O)O)C2(C)C)C(C)(C)C(=O)NC(CC(N)=O)(CC1CCC1)C(=O)O</smiles>
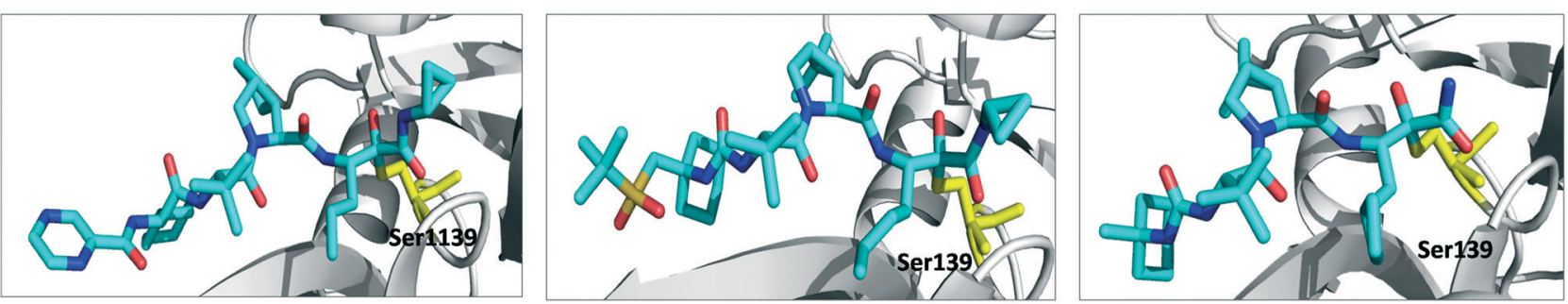

Fig. 6 Chemical structures of covalent inhibitors and their crystal structures with the NS3/4A protease complex (a) telaprevir binding to Ser-1139 (PDB ID 3SV6), (b) narlaprevir (PDB ID 3LON), and (c) boceprevir (PDB ID 3LOX) binding to Ser-139. Inhibitors are shown as cyan sticks and Ser1139, Ser-139 are shown as yellow sticks.

(a)

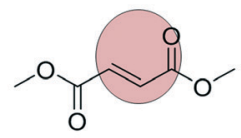

Dimethyl fumarate

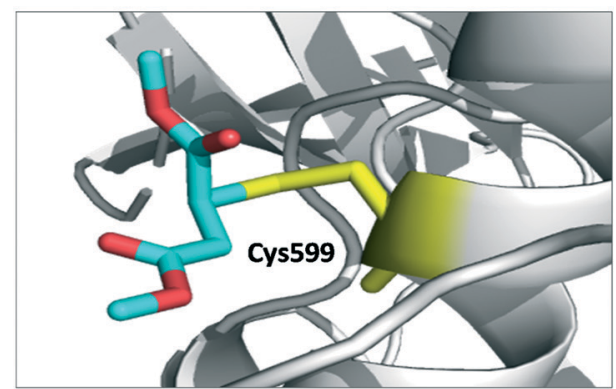

(c)
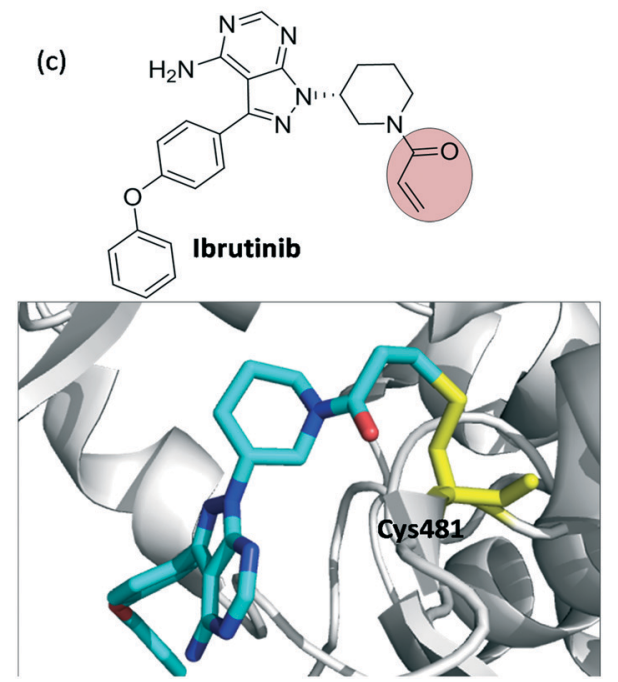

Fig. 7 Chemical structures and binding modes with the target protein: (a) dimethyl fumarate binding to Cys-599 (PDB ID 5O1S), (b) EGFR kinase inhibitor dacomitinib, binding Cys-797 (PDB ID 4I24), (c) ibrutinib (PDB ID 5P9J), and (d) zanubrutinib (PDB ID 6J6M) binding to Cys-481 of BTK. Inhibitors are shown as cyan sticks and Cys-599, Cys-797, Cys-481 are shown as yellow sticks. (b)

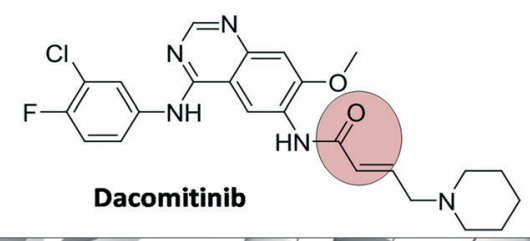

(d)

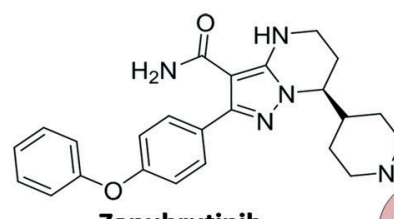

Zanubrutinib

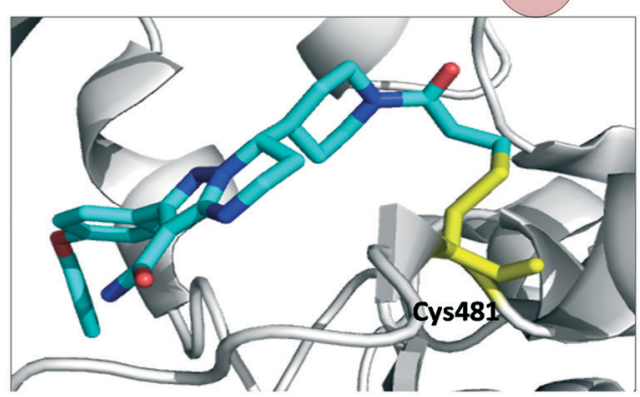


Table 2 Examples of covalent inhibitors in clinical trials

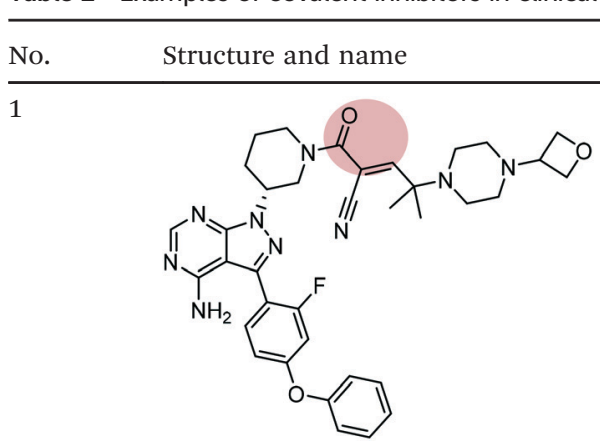

PRN1008

2<smiles>C=CC(=O)N1CCC(CNc2ncnc(N)c2-c2ccc(Oc3ccccc3)cc2)CC1</smiles>

BTK inhibitor

$\alpha, \beta$-Unsaturated carbonyl

III

Evobrutinib

3<smiles>C=CC(=O)N1CCN(c2nc(=O)n3c(nc(N4CCCCC4C)c4cc(F)c(-c5c(O)cccc5F)nc43)c2C)C(C)C1</smiles>

KRAS inhibitor

$\alpha, \beta$-Unsaturated carbonyl

II

AMG510

4<smiles>C/C=C\C(=O)N1CCC(Oc2cc3c(Nc4ccc(Cl)c(Cl)c4F)ncnc3cc2OC)CC1</smiles>

Poziotinib<smiles>C=CC(=O)N1C[C@H](Nc2ncnc3[nH]ccc23)CC[C@@H]1C</smiles>

JAK3 inhibitor

$\alpha, \beta$-Unsaturated carbonyl

II

BTK inhibitor

$\alpha, \beta$-Unsaturated carbonyl

II

Remibrutinib<smiles>C/C=C\C(=O)N(C)CCOc1c(N)ncnc1-c1cc(F)cc(NC(=O)c2ccc(C3CC3)cc2F)c1C</smiles>

7

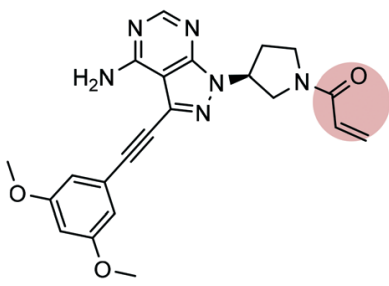

FGFR inhibitor

$\alpha, \beta$-Unsaturated carbonyl

II 
Table 2 (continued)

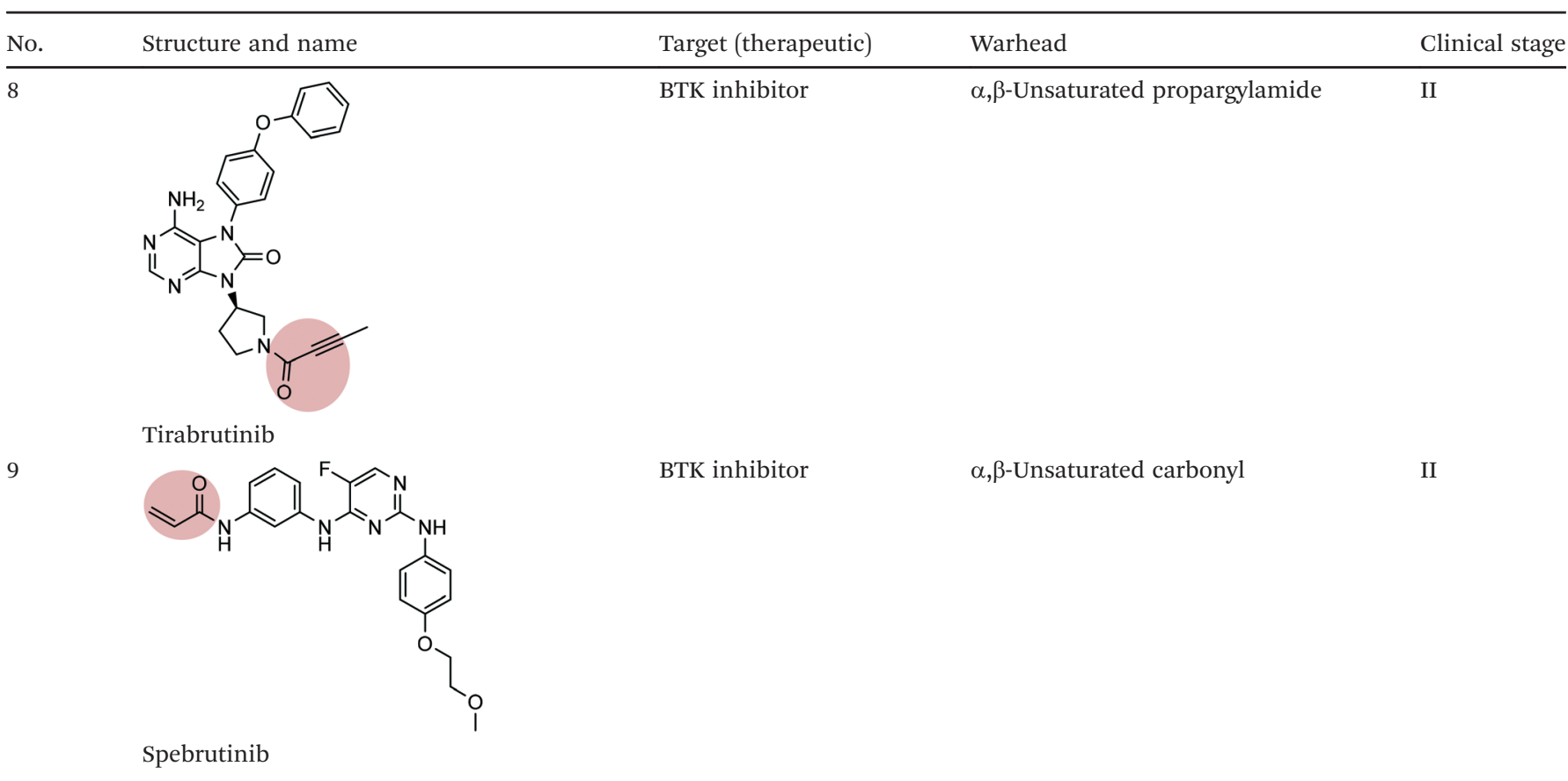

due to less-frequent dosing, (4) reducing the possibility of drug resistance, and (5) targeting shallow binding sites. Each of these advantages is interconnected. For example, since they are highly potent, with low $\mathrm{IC}_{50}$ values, and long binding duration, the necessary dose and intake frequency are lower compared to normal drugs. Indeed, it has been shown that the compliance of patients increases significantly with lower dosing frequency, especially with once-daily dosing, ${ }^{12}$ which can be achieved with a covalent inhibitor. It has also been reported that covalent inhibitors have potential advantages against resistance-prone targets. In 2013, Walter et al. ${ }^{13}$ showed that a novel covalent inhibitor, CO-1686, inhibits a drug-resistance mutant of T790M EGFR. In this case, the warhead is forming a covalent bond to Cys-797.

On the other hand, covalent inhibitors are often considered to have poor selectivity due to their high reactivity. However, several studies have shown that covalent inhibitors are amazingly selective. In a study on quinazoline inhibitors, Zeng et $a .^{14}$ found that a targeted small covalent inhibitor suppresses KRAS G12C, and the selectivity was higher than that of allosteric compounds. Similarly, a study by Kempson et al. ${ }^{15}$ on Janus kinases (JAKs) showed high selectivity against JAK3 due to the addition of a covalent warhead in the compound. The same study also showed that the compound has lower activity towards other JAK family kinases, thus reducing the chance of nonspecific binding. Another example in 2010, Hagel et $a l^{16}$ designed and synthesized small covalent binders towards protease that showed inhibition towards HCV NS3/4A viral protease (HCVP), while having weak reactivity towards other thiols, including glutathione.

Meanwhile, covalent inhibitors have their disadvantages. For example, they (1) may cause unexpected toxicity or hypersensitivity, (2) may cause drug-induced toxicity, (3) may not be suitable for targets that are rapidly turned over/ degraded by enzymes, and (4) may cause problems in choosing the correct warhead. The recently published article regarding covalent warheads may become a guideline, problem, and limitation in developing a covalent warhead, especially when it comes to the actual design, synthesis, and in vivo studies. ${ }^{1}$ However, the increasing amount of knowledge regarding the mechanism of action and the reactivity of many types of warheads may facilitate the future design of covalent inhibitors and assist in tuning their properties to minimize their disadvantages.

\section{Covalent drugs on the market and advanced clinical trials}

Among the covalent inhibitors on the market, about $28 \%$ are used in oncology related targets, $23 \%$ are used in CNS and cardiovascular disorders, $21 \%$ are anti-infectives (mostly the $\beta$-lactam class of antibiotics), and about $11 \%$ are used in gastrointestinal diseases (Fig. 4).

In the last 10 years, there are 14 newly approved covalent drugs (Table 1). However, two of them, telaprevir and boceprevir, were discontinued in 2014 and 2015 respectively due to their low demand compared to newer generation of anti HCV agents. Among these newly approved covalent drugs, there are 9 drugs with $\alpha, \beta$-unsaturated carbonyl as their warhead. Here, a structural analysis of the binding mode for the recently approved covalent inhibitors is provided.

Abiraterone is a drug to treat prostate cancer. Unlike most covalent inhibitors, there is no warhead present in it. Instead, the nitrogen atom from pyridine ring in 
(a)<smiles>[M]C1CN(C(=O)CCCCCCCCC)CCN1c1nc(=O)n(-c2c(C)ccnc2C(C)C)c2nc(-c3c(O)cccc3F)c(F)cc12</smiles>

AMG510

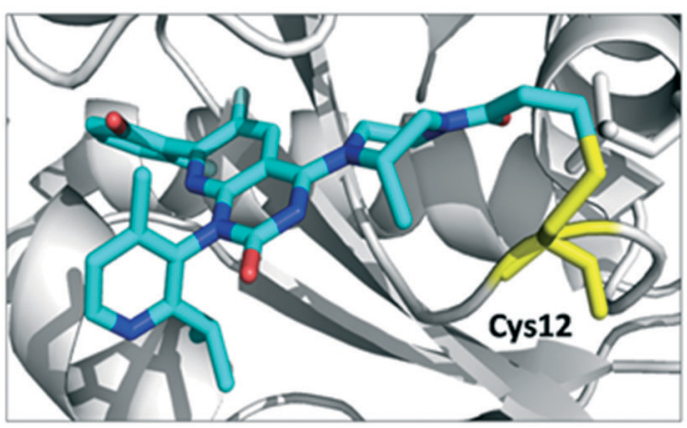

(b)<smiles>C=C(C)C(=O)N1CCN(c2nc(OC[C@@H]3CCCN3C)nc3c2CCN(c2cccc4cccc(Cl)c24)C3)C[C@H]1CC#N</smiles>

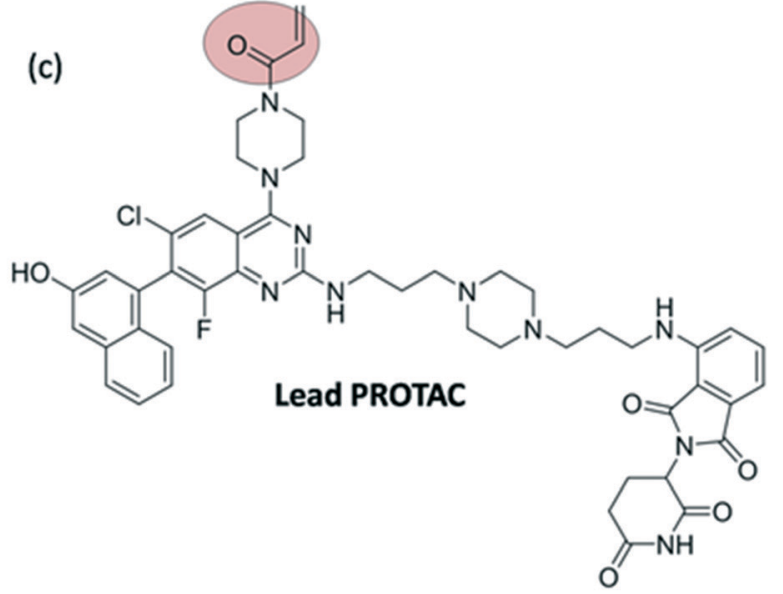

Fig. 8 Chemical structures and binding modes with the target protein: (a) AMG510 (PDB ID 6OIM) and (b) MRTX-849 (PDB ID 6UT0) binding to Cys-12 of KRAS (G12C). Inhibitors are shown as cyan sticks and Cys-12 is shown as yellow sticks, (c) structure of CRBN-KRASG12C lead PROTAC described by Gray et al. ${ }^{27}$<smiles>Nc1ncnc2c1c(-c1ccc(Oc3ccccc3)cc1)nn2C1CC(C(=O)O)N(C(=O)C2C=CCCC2)C1</smiles>

Ibrutinib analogue 1<smiles>Cc1ccc(NC(=O)c2cccc(C(F)(F)F)c2)cc1C(=O)Nc1cnc(Nc2cccc(NC(=O)CNC(=O)C3CC4CCCCC3C4)c2)nc1</smiles>

PLS-123<smiles>Cc1ccc(NC(=O)c2cccc(C(F)(F)F)c2)cc1C(=O)Nc1cnc(Nc2cccc(NC(=O)C(CCC(=O)O)NC(=O)CC3CCCCCC3)c2)nc1</smiles>

PLS-123 analogue 2

Fig. 9 Structure of ibrutinib analogue 1, PLS-123, and PLS-123 analogue 2. 


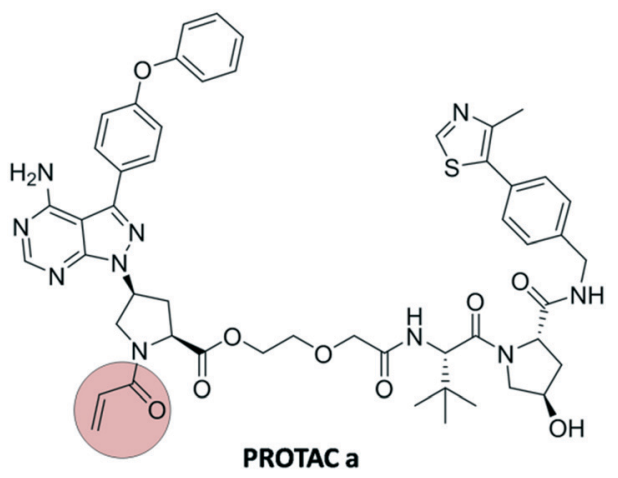

Fig. 10 Structure of PROTAC a targeting BTK.

abiraterone binds covalently to HEM-600 of the enzyme CYP17A1 (Fig. 5). ${ }^{17}$

Telaprevir, narlaprevir and boceprevir are serine protease inhibitors targeting, in particular, the hepatitis C virus NS3/ $4 \mathrm{~A}$ protease. All of them contain the $\alpha$-ketoamide warhead. Telaprevir binds to Ser-1139, whereas narlaprevir and boceprevir bind to Ser-139 (Fig. 6).

Dimethyl fumarate, dacomitinib, and ibrutinib and zanubrutinib are examples of covalent inhibitors containing the $\alpha, \beta$-unsaturated carbonyl warhead (Fig. 7). Dimethyl fumarate is used for the treatment of relapse-remitting multiple sclerosis. It binds to Cys-599 of p90 Ribosomal S6 Kinase. Dacomitinib, ibrutinib, and zanubrutinib are anticancer drugs. Dacomitinib is an EGFR kinase inhibitor to treat non-small cell lung cancer; it binds to Cys-797 of T790M EGFR kinase. Ibrutinib and zanubrutinib are BTK inhibitors used widely to treat mantle cell lymphoma. Both inhibitors bind to Cys-481 of BTK.

Apart from the marketed covalent drugs, there are many more inhibitors currently undergoing clinical trials in phase II or higher. These inhibitors are aiming at cysteine residues of the target proteins (Table 2).

\section{Recent development of covalent inhibitors}

As mentioned before, there is increasing interest in developing covalent inhibitors. Here, we highlight a few applications of covalent inhibitors.

\subsection{RAS}

One of the most notorious oncology targets is still the RAS protein. RAS is a GTPase, transmitting signals for cell growth and division. ${ }^{18}$ For decades RAS was considered an undruggable target. ${ }^{19,20}$ One of the first successful attempts to target the KRAS mutant G12C was described by Shokat et al. involving covalent inhibitors bearing the acrylamide moiety. ${ }^{21}$ After many different attempts to tackle KRAS, in 2018 Amgen developed AMG510, a covalent inhibitor targeting KRASG12C. Notably, AMG510 was the first compound to undergo clinical trials after three decades of research. MRTX-849 (developed by Mirati Therapeutics) is also currently under clinical investigation (Fig. 8). ${ }^{22-24}$ Recently, KRAS was also explored in the field of proteolysis targeting chimeras (PROTACs). PROTACs are an exciting new modality of heterobifunctional molecules aiming to degrade the protein of interest instead of inhibiting it. ${ }^{25,26}$ In 2019, Gray et $a .^{27}$ reported the development of a cereblon-based degrader molecule library targeting KRASG12C and bearing acrylamide warheads. Although the lead PROTAC (Fig. 8c) of the series showed cereblon engagement in cells and bound to KRASG12C in vitro, it was unable to effectively polyubiquitinate endogenous KRAS and thus degradation was not observed in pancreatic and lung cancer cells. Further studies will be necessary to fully understand the challenges of targeting KRAS using PROTACs.

\subsection{BTK and PROTACs}

In the last decade, several covalent inhibitors targeting Bruton's tyrosine kinase (BTK) were discovered, including ibrutinib and zanubrutinib. These compounds contain an acrylamide warhead, targeting Cys-481 in the ATP binding domain of BTK. Recently, these covalent kinase inhibitors were further developed as PROTACs. In 2019, Xue et al. ${ }^{28}$ developed a series of PROTACs bearing covalent warheads. Ibrutinib and PLS-123 as covalent inhibitors were chosen as the backbone of the modified compounds 1 and 2 (Fig. 9).

The analogues 1 and $\mathbf{2}$ were then combined with ligands targeting E3 ligases, either pomalidomide or VH032, through a series of linkers and their activity towards BTK was examined. The active group of acrylamide was also replaced with propanamide as comparison towards covalent vs. noncovalent inhibition. The combination between analogue 1 (ibrutinib analogue) and VH032 (PROTAC a, Fig. 10) showed excellent results regarding BTK degradation compared to other combinations and non-covalent PROTACs, ${ }^{27}$ highlighting a new application of covalent warheads in protein degradation.

\section{Conclusions}

The rational development of covalent inhibitors has been steadily increasing in the past decades. Numerous warheads have been developed, expanding the covalent warhead toolbox and allowing for selective targeting of specific amino acid residues. More than 50 covalent compounds are on the market or in advanced clinical trials. As more data emerge regarding safety and efficacy of covalent drugs, the structureguided optimization and rational development will be facilitated in the future. Thus, covalent inhibitors remain an attractive alternative for difficult targets, including proteinprotein interactions, where selectivity may remain elusive with non-covalent inhibitors. Moreover, the recent examples of PROTACs bearing covalent warheads show their potential in new modalities, whereas the development of diverse covalent warheads allows for careful tuning of their reactivity and selectivity. 


\section{Conflicts of interest}

There are no conflicts to declare.

\section{Acknowledgements}

This research has been supported (to AD) through ITN "Accelerated Early stage drug dIScovery" (AEGIS, grant agreement No. 675555), the National Institute of Health (NIH) (2R01GM097082-05), the European Lead Factory (IMI) (grant agreement number 115489), the Qatar National Research Foundation (NPRP6-065-3-012) and COFUNDs ALERT (grant agreement No. 665250), Prominent (grant agreement No. 754425) and KWF Kankerbestrijding grant (grant agreement No. 10504). Fandi Sutanto acknowledges Indonesian Endowment Fund for Education (Lembaga Pengelola Dana Pendidikan) for financial support.

\section{Notes and references}

1 M. Gehringer and S. A. Laufer, J. Med. Chem., 2018, 62, 5673-5724.

2 J. Singh, R. C. Petter, T. A. Baillie and A. Whitty, Nat. Rev. Drug Discovery, 2011, 10, 307-317.

3 R. A. Bauer, Drug Discovery Today, 2015, 20, 1061-1073.

4 T. Shimokawa and W. Smith, J. Biol. Chem., 1992, 267, 12387-12392.

5 D. J. Waxman and J. L. Strominger, Annu. Rev. Biochem., 1983, 52, 825-869.

6 J. M. Shin, K. Munson, O. Vagin and G. Sachs, Pflugers Arch, 2009, 457, 609-622.

7 K. Munson, R. Garcia and G. Sachs, Biochemistry, 2005, 44, 5267-5284.

8 E. Fellenius, T. Berglindh, G. Sachs, L. Olbe, B. Elander, S.-E. Sjöstrand and B. Wallmark, Nature, 1981, 290, 159-161.

9 A. S. Michalopoulos, I. G. Livaditis and V. Gougoutas, Int. J. Infect. Dis., 2011, 15, e732-e739.

10 E. D. Brown, E. I. Vivas, C. T. Walsh and R. Kolter, J. Bacteriol., 1995, 177, 4194-4197.

11 I. Kola and J. Landis, Nat. Rev. Drug Discovery, 2004, 3, 711-716.

12 C. I. Coleman, B. Limone, D. M. Sobieraj, S. Lee, M. S. Roberts, R. Kaur and T. Alam, J. Manage. Care Pharm., 2012, 18, 527-539.
13 A. O. Walter, R. T. T. Sjin, H. J. Haringsma, K. Ohashi, J. Sun, K. Lee, A. Dubrovskiy, M. Labenski, Z. Zhu and Z. Wang, Cancer Discovery, 2013, 3, 1404-1415.

14 M. Zeng, J. Lu, L. Li, F. Feru, C. Quan, T. W. Gero, S. B. Ficarro, Y. Xiong, C. Ambrogio and R. M. Paranal, Cell Chem. Biol., 2017, 24, 1005-1016. e1003.

15 J. Kempson, D. Ovalle, J. Guo, S. T. Wrobleski, S. Lin, S. H. Spergel, J. J.-W. Duan, B. Jiang, Z. Lu and J. Das, Bioorg. Med. Chem. Lett., 2017, 27, 4622-4625.

16 M. Hagel, D. Niu, T. St Martin, M. P. Sheets, L. Qiao, H. Bernard, R. M. Karp, Z. Zhu, M. T. Labenski and P. Chaturvedi, Nat. Chem. Biol., 2011, 7, 22-24.

17 M. Fernández-Cancio, N. Camats, C. E. Flück, A. Zalewski, B. Dick, B. M. Frey, R. Monné, N. Torán, L. Audí and A. V. Pandey, Pharmaceuticals, 2018, 11, 37.

18 D. S. Goodsell, Stem Cells, 1999, 17, 235-236.

19 A. D. Cox, S. W. Fesik, A. C. Kimmelman, J. Luo and C. J. Der, Nat. Rev. Drug Discovery, 2014, 13, 828-851.

20 J. Downward, Nat. Rev. Cancer, 2003, 3, 11-22.

21 J. M. Ostrem, U. Peters, M. L. Sos, J. A. Wells and K. M. Shokat, Nature, 2013, 503, 548-551.

22 J. Hallin, L. D. Engstrom, L. Hargis, A. Calinisan, R. Aranda, D. M. Briere, N. Sudhakar, V. Bowcut, B. R. Baer and J. A. Ballard, Cancer Discovery, 2020, 10, 54-71.

23 ClinicalTrials.gov Identifier: NCT03600883. A Phase 1/2, Study Evaluating the Safety, Tolerability, PK, and Efficacy of AMG 510 in Subjects With Solid Tumors With a Specific KRAS Mutation (CodeBreak 100), 2018, Available from: https:/clinicaltrials.gov/ct2/show/ NCT03600883.

24 J. Canon, K. Rex, A. Y. Saiki, C. Mohr, K. Cooke, D. Bagal, K. Gaida, T. Holt, C. G. Knutson and N. Koppada, Nature, 2019, 575, 217-223.

25 M. Konstantinidou, J. Li, B. Zhang, Z. Wang, S. Shaabani, F. Ter Brake, K. Essa and A. Dömling, Expert Opin. Drug Discovery, 2019, 14, 1255-1268.

26 M. Scudellari, Nature, 2019, 567, 298.

27 M. Zeng, Y. Xiong, N. Safaee, R. P. Nowak, K. A. Donovan, C. J. Yuan, B. Nabet, T. W. Gero, F. Feru, L. Li, S. Gondi, L. J. Ombelets, C. Quan, P. A. Jänne, M. Kostic, D. A. Scott, K. D. Westover, E. S. Fischer and N. S. Gray, Cell Chem. Biol., 2020, 27, 19-31. e16.

28 G. Xue, J. Chen, L. Liu, D. Zhou, Y. Zuo, T. Fu and Z. Pan, Chem. Commun., 2020, 56, 1521-1524. 